\title{
Comunicación

\section{La concentración en el mercado de la televisión restringida en México}

\author{
RODRIGO GÓMEZ1
}

GABRIEL SOSA2

Este trabajo analiza el estado que presenta la industria de la televisión restringida en México. El principal objetivo de esta investigación es, por un lado, generar información estadística que permita medir la concentración económica y de mercado del subsector de cara a los cambios que está planteando la convergencia tecnológica y el triple play (voz, video e Internet en una sola red o infraestructura de trasmisión); por el otro, problematizar sobre sus consecuencias socioculturales. Así mismo se presenta un breve recorrido histórico del subsector para entender con mayor profundidad las particularidades del caso mexicano.

PALABRAS CLAVE: concentración, industrias culturales, televisión de paga, telecomunicaciones, convergencia.
This paper analyzes the market of the pay television industry in Mexico. The main objective of this research is, firstly, to generate statistical information to measure the economic and market concentration of the sub-sector in the face of changes that are being established with the technological convergence and triple play (voice, video and Internet in one network and transmission infrastructure). It also presents a brief historical review of the subsector to understand more fully the particularities of the Mexican case.

KEY WORDS: concentration, convergence cultural industries, pay television, telecommunications.

1 Universidad Autónoma Metropolitana-Cuajimalpa, México.

Correo electrónico: rgg28jaguar@gmail.com

2 Universidad Autónoma Metropolitana-Xochimilco, México.

Correo electrónico: gsosap@yahoo.com 


\section{INTRODUCCIÓN}

La televisión de paga es el sector televisivo que más ha crecido en los últimos años, al surgir empresas y plataformas que ofrecen este servicio en los distintos estados de la República Mexicana. Este desarrollo se debe a tres circunstancias que van íntimamente interrelacionadas. La primera es de índole política. En efecto, desde principios de los años noventa el gobierno federal ha intensificado el impulso de políticas a través de la neoregulación con el objetivo de permitir la participación de la inversión privada nacional y extranjera. La segunda es de orden económico, puesto que la situación anterior ha propiciado inversiones significativas que han generado fusiones y alianzas de capital entre empresas al presentarse mercados atractivos para su explotación y desarrollo de negocios que han repercutido en jugosas utilidades. Estas fusiones y alianzas no sólo se generaron entre operadores de los sistemas de televisión de paga, sino que han involucrado a empresas que ya tenían presencia en el mercado de la televisión abierta (particularmente Grupo Televisa). Y la tercera es la que tiene que ver con el desarrollo de las nuevas tecnologías y la convergencia entre los medios de comunicación y las telecomunicaciones que les permiten ofrecer un mayor número de servicios (Crovi, 2005). ${ }^{3}$

La administración del presidente Felipe Calderón (2006-2012) ha colocado al sector de las telecomunicaciones $-\mathrm{y}$ a la televisión de paga como parte de dicho sector- como uno de los ejes fundamentales de desarrollo económico, en virtud de sus elevados índices de crecimiento comparados con otras actividades productivas. ${ }^{4}$ De manera específica, el Programa Sectorial de Comunicaciones y Transportes 2007-2012, tiene como meta "promover la competencia entre concesio-

3 Actualmente los sistemas de televisión de pago, gracias a la digitalización, pueden ofrecer una mejor resolución y recepción de imagen y sonido, así como una oferta amplia de canales. También pueden prestar otro tipo de servicios de valor agregado como Internet, pago por evento, telecompra, telefonía fija, entre otras.

4 El subsector de las comunicaciones ha sido el más dinámico del sector y uno de los más importantes de la economía, habiendo crecido en promedio $14.9 \%$ entre 1997 y 2007, lo que equivale a casi cinco veces el crecimiento de la economía en su conjunto (SCT, 2007). 
narios a fin de ampliar la cobertura de los servicios del país y lograr que las tarifas permitan el acceso a un mayor número de usuarios". Esto se logrará precisa, "promoviendo el desarrollo de infraestructura tecnológica de conectividad que permita alcanzar una mayor penetración de la población desde cualquier rincón del país" (SCT, 2007, p. 11).

En el caso específico de la televisión restringida el objetivo para 2012 es llegar a los 10 millones de suscriptores (SCT, 2007, p. 15). Cuando se elaboró el Programa, se consideraron 6 millones de suscriptores en las diferentes plataformas tecnológicas disponibles para este servicio. Al término de 2008 ya se había sumado otro millón de suscriptores para alcanzar más de 7 millones. Para el tercer trimestre de 2009, se contaba con 7’613,000 suscriptores, como se detallará más adelante (Cofetel, 2009).

\section{MÉTODO PARA MEDIR LA CONCENTRACIÓN}

En la actualidad existen varios métodos de corte económico que nos permiten medir la concentración de los distintos sectores económicos e industriales (De Miguel, 1993; Hoskins, McFadyen \& Finn, 2004). En el caso de las industrias culturales o de las industrias mediáticas, ésta ha sido una de las principales preocupaciones de los especialistas en el estudio de los medios de comunicación, pues se parte de la premisa que entre mayor concentración de industrias culturales, existe una limitación en la diversidad de los contenidos que produce este particular sector (Iosifides, 1997; Sánchez, 2006). Es decir, no sólo se plantean problemáticas de mercado, de oferta y demanda, sino que también la concentración puede reflejar falta de pluralidad de información e inhibición de las distintas expresiones culturales que existen en una sociedad determinada.

Uno de los objetivos de esta investigación es medir la concentración del mercado de la televisión de paga. Para esto se utiliza el índice de concentración de las cuatro empresas dominantes (CR4 -radio de concentración-), donde se mide el coeficiente de facturación y el coeficiente de audiencia. En esta primera etapa los dos indicadores considerados son las ganancias totales empresas y los abonados, ${ }^{5}$ respectivamente. Por

5 La metodología de análisis se inspira en la propuesta de Becerra y Mastrini (2006) de medición de la concentración de las distintas industrias culturales 
ejemplo, el CR4 mide el porcentaje de la cuota de mercado que acumulan las cuatro empresas o firmas dominantes. La regla es que la industria es altamente concentrada si el CR4 es igual o mayor a 50\% (oligopolio), moderadamente concentrado si el CR $4=34 \%$ a $49 \%$ (competencia monopólica), y bajamente concentrado si el CR4 es igual o menor a $33 \%$ (competencia perfecta) (Hoskins et al., p. 146).

Un aspecto que debemos considerar como punto de partida para entender el tema es la relación histórica-estructural que ha existido entre los distintos empresarios dominantes que han participado en el subsector con el sistema político mexicano, lo cual explica de forma importante las lógicas anticompetitivas que prevalecen en la televisión de paga. La falta de mecanismos democráticos y de rendición de cuentas por parte de los distintos gobiernos de cara a la sociedad, sobre todo al momento de otorgar las concesiones e implementar y ejecutar leyes y reglamentos a modo de los intereses empresariales, derivó en una estructura oligopólica. Así mismo debemos enmarcar estas circunstancias en una dinámica capitalista orientada y articulada por la mercantilización de los bienes y servicios comunicativos y culturales (Mosco, 1996; Murdock, 2006).

La televisión de paga se ofrece hasta ahora por tres diferentes sistemas: el cable, las microondas punto multipunto (en inglés Multipoint Multichannel Distribution Service, MMDS) y vía satélite o DTH (Direct To Home).

\section{COORDENADAS TEÓRICO-METODOLÓGICAS}

La concentración en las distintas industrias culturales y de la información es una característica de nuestros tiempos (Becerra \& Mastrini, 2006; Boquillon, Miège \& Moriset, 2006; Bustamante, 2003; Garnham, 2000), no obstante la retórica neoliberal nos dice que los mercados deben ser competitivos para poder ofrecer los mejores precios y estándares de calidad para los consumidores, así como la suficiente pluralidad para acceder al amplio "mercado de las ideas". Sin embargo, en la práctica, promueven los oligopolios y monopolios, "en donde

en América Latina, para presentar de forma sencilla la concentración de los mercados de las industrias culturales y de la información. 
un pequeño número de conglomerados o firmas acumulan el suficiente poder en el mercado para manipular a éste en su favor y a expensas del consumidor-ciudadano" (Garnham, 2000, p. 54).

La problemática de la concentración ocupa las miradas tanto de inversionistas nacionales como extranjeros, así como de los organismos supranacionales -principalmente en la Unión Europea- por sus implicaciones en la distorsión de los mercados, pero también llama la atención de aquellos preocupados en la "credibilidad" de los medios, la "calidad" de la producción cultural e informativa y finalmente de la "diversidad" de los contenidos culturales (Miège, 2006, p. 155). Es decir, la concentración de capital y de los mercados culturales: a) inhibe la competencia, b) pone en entredicho la pluralidad y credibilidad de la información de las industrias mediáticas, c) obstaculiza la diversidad de los productos culturales y d) deteriora la calidad de éstos al no tener contrapesos y espacios suficientes para la innovación y experimentación creativa.

Otro punto de partida de nuestra investigación, coincidiendo con los investigadores y especialistas en la "economía política de la comunicación y la cultura" como Peter Golding, Graham Murdock, Vincent Mosco y Bernard Miège, es que la observación de la concentración y el tamaño de las empresas como las analizadas en este escrito son solamente los puntos de partida para comprender las distintas transformaciones del negocio de las industrias culturales y de la información (Golding \& Murdock, 2000; Miège, 2006; Mosco, 1996).

Siguiendo a Bernard Miège (2006), consideramos que en la actualidad la concentración debe ser pensada de forma amplia y en un contexto global, "porque está compuesta de movimientos múltiples y además porque encubre fenómenos menos fáciles de interpretar que no se ven a simple vista..." (p. 159). Aquí Miège se refiere a las consecuencias que se dan en la producción cultural e informacional, sobre todo porque éstas no pueden deducirse mecánicamente. Si bien esta investigación no se ocupa de este aspecto en profundidad, nos parece importante considerarlo para un trabajo que continúe en esa dirección.

En esa misma línea es importante cuestionar, ¿cómo está afectando Internet el suministro de la información basada en los bienes culturales? (Hoskins et al., 2004, p. 1) o por lo menos estamos concientes que 
Internet está transformando las formas tradicionales de distribución de los bienes (tangibles) y servicios (intangibles) culturales, lo que tiene implicaciones no sólo económicas en términos de la cadena de valor de los productos o de las estrategias industriales y los formatos audiovisuales, sino también socioculturales y comunicativas en términos de nuevas formas de consumo cultural y comunicacional.

También coincidimos con Nicholas Granham (2005) cuando dice "que el centro de gravedad de la economía capitalista está cambiando de la producción y circulación de los bienes materiales a la produción y circulación de los valores y bienes simbólicos" (p. 55). De ahí la importancia de observar a un subsector clave de las industrias culturales y de la información que está en mutación y que se está convirtiendo en uno de los principales proveedores de los bienes y servicios informacionales, comunicativos y culturales.

\section{ORÍGENES Y CONTENIDOS}

\section{Televisión por cable}

La televisión por cable tuvo entre sus principales promotores a familias y capitales cercanos al Grupo Televisa a través de dos vías: la primera por medio de Cablevisión, empresa que comenzó a ofrecer el servicio de televisión por cable desde 1968 en el Distrito Federal; la segunda, bajo el nombre de Intermex y la dirección del Ing. Benjamín Burillo, ${ }^{6}$ al construirse las instalaciones de cableado y redes de conexión para diversos sistemas de televisión por cable en el norte del país. ${ }^{7}$

En sus inicios este sistema fue dirigido principalmente al consumo de las clases medias y altas que buscaban programación de origen estadounidense $\mathrm{o}$, en su defecto, a extranjeros de esa nacionalidad que deseaban seguir vinculados de alguna forma a su país de origen (Sevilla, 1998). En menor medida el servicio se comercializó con la idea de alcanzar a comunidades o regiones en donde no llegaba la televisión en abierto na-

6 Este personaje estuvo casado con la hija de Emilio Azcárraga Vidaurreta, fundador de Televisa.

7 Para una revisión detallada sobre los orígenes del sector en México se recomienda revisar a Sevilla (1998) y a Crovi $(1999,2005)$. 
cional por razones técnicas. De cualquier manera, este subsector se ha caracterizado sólo por su función de distribuidor de señales, principalmente de origen estadounidense (Crovi, 2005) y sólo durante la última década ha comenzado a incorporase en la producción audiovisual mexicana.

La mayoría de los concesionarios del interior de la República formaron la Productora y Comercializadora de Televisión (PCTV) 8 en 1989 para negociar en bloque diversos servicios como la compra de señales extranjeras que ofrecen sus distintos sistemas, la renta de transponders de satélites y la creación de una casa productora de contenidos (Sevilla, 1998; Crovi, 1999). ${ }^{9}$ Hasta 2008 PCTV se encargaba de la programación de seis canales que son: TVC, de perfil generalista; TVC Platino y TVC Platino Plus, cine de estreno; Cine Mexicano por Cable (CMC); TVC Deportes, canal deportivo, y Cine Nostalgia y ARTVC, dedicado a exhibir cine de arte. Además, estableció un convenio con la cadena estadounidense especializada en programación deportiva ESPN ${ }^{10}$ para coproducir la señal de ESPN 2 Latino, que se distribuye en toda Latinoamérica. En 2008 la empresa anunció la firma de un convenio con Fox Sports Latin America para establecer una alianza técnica y operativa para que México sea su centro de producción, con la finalidad de incrementar el número de programas mexicanos de sus distintos formatos que se han colocado de forma importante en los televidentes hispanos en Estados Unidos y, claro, para bajar los costos de producción del conglomerado, pues antes el centro de operaciones de dicha filial de Fox estaba en Los Ángeles.

Otro aspecto que llama la atención de PCTV es que ha iniciado ya un proceso de expansión internacional y se encuentra comercializando sus señales de producción propia en Centroamérica y en breve pretenden cubrir la totalidad de los mercados de América Latina. En este sentido

8 Esta empresa en 1999 agrupó 187 sistemas, lo que representaba 96\% del sector (Crovi, 1999).

9 Esta situación era clave para las empresas, ya que por ley se establece que para poder comercializar sus espacios deben generar producción propia. De esta forma podían obtener recursos diferentes a los provenientes de los servicios directos al usuario.

10 Esta empresa, creada a finales de los años setenta, actualmente filial de Disney-ABC, fue el primer canal 100\% deportivo. 
se advierte su posibilidad de expansión internacional a partir de sus ventajas competitivas ante mercados en desarrollo.

En suma, PCTV produce seis señales de televisión; tiene contratados con cinco proveedores los derechos para comercializar de manera exclusiva 14 señales de televisión y con otros proveedores los derechos para transmitir de manera no exclusiva otras 40 señales más. En total, son 59 señales las que comercializa con los operadores de televisión por cable. ${ }^{11}$

Estos movimientos en bloque de los empresarios de la televisión por cable del interior del país, a través de empresas como PCTV, hace algunos años buscaban hacer frente al dominio del Grupo Televisa en cuanto a la distribución de señales nacionales y extranjeras, y a la competencia que representaba Multivisión con su sistema MMDS en varias ciudades del país. Sin embargo, como veremos más adelante, esta situación cambió al convertirse Grupo Televisa, también, en el principal operador de la televisión de paga. También, con PCTV, sus miembros se protegían de la competencia en el mismo subsector, al llevar a cabo prácticas monopólicas con nuevos concesionarios, lo que llevó a la Comisión Federal de Competencia (CFC) a sancionar a dicha productora-comercializadora. ${ }^{12}$

\section{Televisión MMDS}

En 1989, la entrada de Multivisión en la competencia de la televisión de paga con una nueva tecnología, MMDS, generó que el sector entrara en

11 Entre éstos destacamos las señales de la productora de Discovery Chanel, National Geographic, Hallmark y Nickelodeon (Telemundo, 2008).

12 PCTV negó la provisión de señales de televisión a diversos concesionarios de televisión por cable, denominados "segundos concesionarios" en el país, con el objetivo de crear ventajas exclusivas a favor de los operadores socios de esa empresa, más conocidos como "primeros concesionarios". Es decir, se buscaba otorgar privilegios a quienes se establecían primero en una plaza determinada y obstaculizaban la contratación de sus señales a quienes competían en el mismo mercado. La CFC recibió diversas denuncias y estudió los casos. Mientras lo hacía, PCTV se comprometió ante dicho órgano a dar acceso no discriminatorio (must offer) a cualquier concesionario, sea o no miembro o accionista de PCTV, a las señales que produce y distribuye (CFC, 2006). 
una dinámica de constante cambio, tanto en los precios de suscripción y renta mensual, como en la oferta de señales y servicios (por ejemplo, el pago por visión, PPV por sus siglas en inglés), pero sobre todo lo que se logró fue un mayor acceso a estos servicios al tenderse más redes de telecomunicaciones, gracias a las posibilidades de la multidifusión inalámbrica. Hasta 2009, Multivisión era considerado el operador de MMDS más importante del mundo. ${ }^{13}$

Sin embargo, algunas de las concesiones otorgadas para el servicio no habían sido refrendadas hasta febrero de 2010 por el gobierno mexicano, ya que parte de los $190 \mathrm{Mhz}$ concesionados en el segmento de los 2,500 a los 2,700 Mhz los pretendía recuperar para otorgarlos a nuevos operadores y prestar servicios de nueva generación o de $3 \mathrm{G}$ y no sólo de televisión.

En 2008, Multivisión comenzó a prestar en algunas ciudades de México un nuevo servicio de DTH, de bajo costo y con una oferta limitada de canales (el paquete básico es de 15 canales), en una alianza con las estadounidenses Dish y Echostar. Para reforzar su comercialización firmó una alianza estratégica con el gigante de las telecomunicaciones en México, Telmex, para que su servicio de DTH pudiera ser facturado en el recibo telefónico. Esta situación ha generado controversias importantes ante la Comisión Federal de Telecomunicaciones (Cofetel) y CFC impulsadas por la Cámara Nacional de la Industria de las Telecomunicaciones por Cable (Canitec) argumentando que la posición monopólica de Telmex debería impedirle cualquier tipo de alianza con concesionarios de televisión de paga.

13 En efecto, con base en las estadísticas de la Cofetel disponibles en su sitio web (www.cofetel.gob.mx), 90\% del espectro asignado en el país en la banda de frecuencias de los 2.5 a los 2.7 Ghz lo explota en exclusiva MVS; el resto, otras cuatro empresas. Por ello, el grupo es considerado el operador de MMDS más grande en el mundo. Estas licencias abarcan $190 \mathrm{Mhz}$, de los cuales sólo 90 son utilizados actualmente para el servicio de MasTV. Los 100 restantes son explotados para otros servicios. MVS Net, división de MVS Comunicaciones, brinda servicios bidireccionales y de transporte de señales de voz a empresas como Avantel, Alestra, Marcatel y Protel. También MVS desarrolló un servicio propio de Internet denominado E-go. 
Uno de los objetivos de Multivisión con su nuevo servicio de DTH es migrar a sus abonados de la televisión por microondas a la nueva plataforma satelital y desarrollar en la banda terrestre un servicio nacional de banda ancha (WiMax). Sin embargo, su proyecto podría truncarse si, como mencionamos antes, el gobierno decide no renovar sus licencias. Hasta la terminación de este artículo, el asunto se dirimía a través de diversos recursos jurídicos presentados por la empresa.

\section{Televisión DTH}

En 1995, las empresas Hughes Communications, de Estados Unidos; Organización Cisneros, de Venezuela; Televisión Abril, de Brasil y MVS, de México, crean el consorcio Galaxy Latin America para lanzar el servicio de DTH en la región latinoamericana. Este producto surge como parte del proyecto de expansión de Hughes Communications, que un año antes ya ofrecía DTH en Estados Unidos bajo la filial DirecTV. En 1996, DirecTV América Latina, inicia operaciones. Hughes participa con $60 \%$ de la inversión; Organización Cisneros con $20 \%$, en tanto que Televisión Abril y MVs lo hacen cada una con 10\%.

En el mismo año de 1996 se lanza el otro servicio de DTH, Sky, por iniciativa de Televisa, el conglomerado estadounidense News Corporation, la brasileña O'Globo y Tele Communications Internacional Inc. (TCI), que aglutina a sistemas de cable en Estados Unidos. Al igual que DirecTV, Sky es resultado de la expansión internacional de News Corporation. Para el caso latinoamericano, Televisa, News Corporation y O'Globo aportan cada una 30\% de la inversión, mientras que TCI participa con $10 \%$ restante.

Desde entonces, ambas empresas compitieron intensamente en la región, pero en el camino DirecTV asumió fuertes deudas. La exclusividad de la Copa de Futbol de 2002 agravó su situación. Dos años después la empresa decidió cerrar operaciones en México. Sky compró la base de suscriptores de su competidor. El 11 de octubre de 2004, DirecTV explicó que era muy problemático crecer en un mercado donde el acceso de los canales locales (los de Televisa) estaba restringido. El monopolio de la televisión satelital por parte de Sky terminó, como ya se dijo, con el lanzamiento de Dish en México en 2008. 


\section{Contenidos}

En materia de contenidos, el cable, el MMDS y después el DTH, reproducen y consolidan la tendencia mundial en la especialización y segmentación, es decir, la oferta está determinada en función de canales temáticos, en contraste con la televisión en abierto caracterizada por ser de corte generalista. En el caso de México, dichas señales son mayoritariamente de origen estadounidense. Este último elemento es una de las asignaturas pendientes de los distintos sistemas de televisión de paga, ya que no se ha incentivado de forma decidida la producción de canales propios, o por lo menos cuotas de producción por señal extranjera, lo que ayudaría en todos los sentidos a la industria audiovisual mexicana, al generar fuentes de empleo y, sobre todo, contenidos que reflejaran las realidades locales y culturales de los distintos estados de la República.

Un estudio realizado a principios de esta década, por la Procuraduría Federal del Consumidor (Profeco), estableció que de los 489 canales que se comercializan en la televisión de paga en México, 27.4\% son de corte musical, $26.8 \%$ de películas y de pago por evento; mientras que los canales de cultura, noticias y servicios representan sólo 9\% (Profeco, 2001).

Finalmente, pensamos ante lo expuesto en este apartado, retomando la idea de Hernández y Orozco (2007), que históricamente en México no se ha fomentado ni impulsado la competencia en los distintos sectores del mercado televisivo, es decir, tenemos una experiencia histórica de no competencia en el sector.

\section{CRECIMIENTO Y MARCO LEGAL}

La televisión de paga en México tuvo un desarrollo importante luego de la publicación de la Ley Federal de Telecomunicaciones en 1995. Esta regulación, de una clara tendencia neoliberal, permitió la inversión extranjera en todo el sector hasta en 49\% (con excepción de la telefonía celular, donde el porcentaje es de 100). ${ }^{14}$ De igual manera estableció los

14 Dos años antes de la publicación de la Ley Federal de Telecomunicaciones, el presidente Carlos Salinas de Gortari publicó el "Decreto por el que se reforman los artículos 10, 33 y 42 del Reglamento del Servicio de Televisión por 
criterios para que empresas de telecomunicaciones, como la televisión por cable o la televisión MMDS, pudiesen convertirse en redes públicas de telecomunicaciones, lo que les dio la posibilidad, desde entonces, de prestar no sólo el servicio de video sino también otro tipo de ofertas a través de sus infraestructuras. La Ley Federal de Telecomunicaciones es considerada como la primera legislación convergente en México y la caracterizamos como una legislación orientada, principalmente, en beneficiar las oportunidades de negocio, soslayando aspectos de orden social y cultural, pese a la incorporación de conceptos como "cobertura social" (Gómez \& Sosa, 2006).

\section{GRÁFICA 1}

DESARROLLO DE LOS ABONADOS DE LOS DISTINTOS SISTEMAS DE TELEVISIÓN DE PAGA DE 1994 A 2008

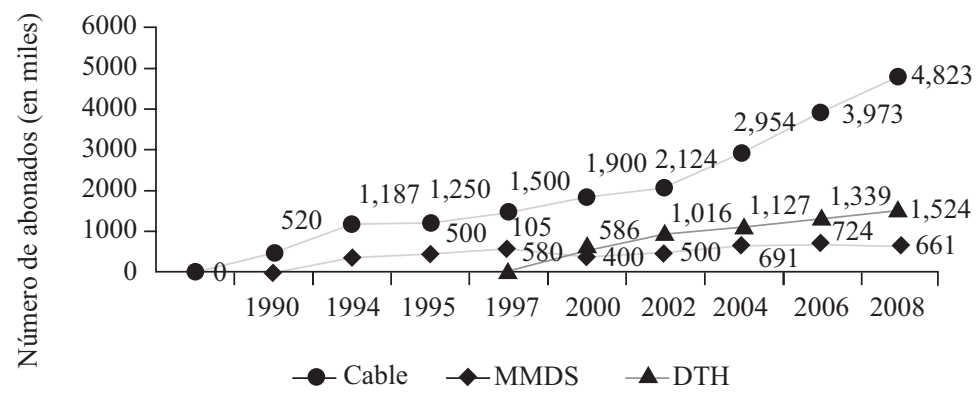

Fuente: Elaboración propia con datos de la Cofetel (2008).

Para apreciar con más claridad el impacto de la ley basta con comentar que antes de su entrada en vigor se habían otorgado sólo cuatro conce-

Cable", mediante el cual se permitió desde entonces la inversión extranjera en la televisión por cable hasta en $49 \%$, acorde con los cambios llevados a cabo en la Ley para Promover la Inversión Mexicana y Regular la Inversión Extranjera, donde se admitió la participación de capital extranjero por el mismo porcentaje en el caso de las empresas prestadoras de servicios de telecomunicaciones (Diario Oficial de la Federación, 1993, 23 de agosto, p. 49). 
siones. Después de su expedición el crecimiento fue importante: en 1996 se otorgaron 117 y para 2000 un total de 219 (Cofetel, 2003). Al mismo tiempo el flujo de inversiones se incrementó de manera sustancial. Por ejemplo, en 1995 se invirtieron 64 millones de dólares; para 2001 esta cifra ascendió a 451 millones de dólares, y en 2008 la cifra rondó los 700 millones de dólares. ${ }^{15}$ En concordancia sus ganancias muestran una tendencia a la alza, pues en 1998 reportaron 350 millones de dólares, en 2001 llegaron a 820 millones de dólares y para 2007 la cifra superaba los 1,200 millones. En términos económicos el balance ha sido claramente positivo en términos de inversión y generación de ingresos.

GRÁFICA 2

CRECIMIENTO DE LOS ABONADOS EN LAS DISTINTAS PLATAFORMAS DE TELEVISIÓN DE PAGA 1990-2008 (EN MILES)

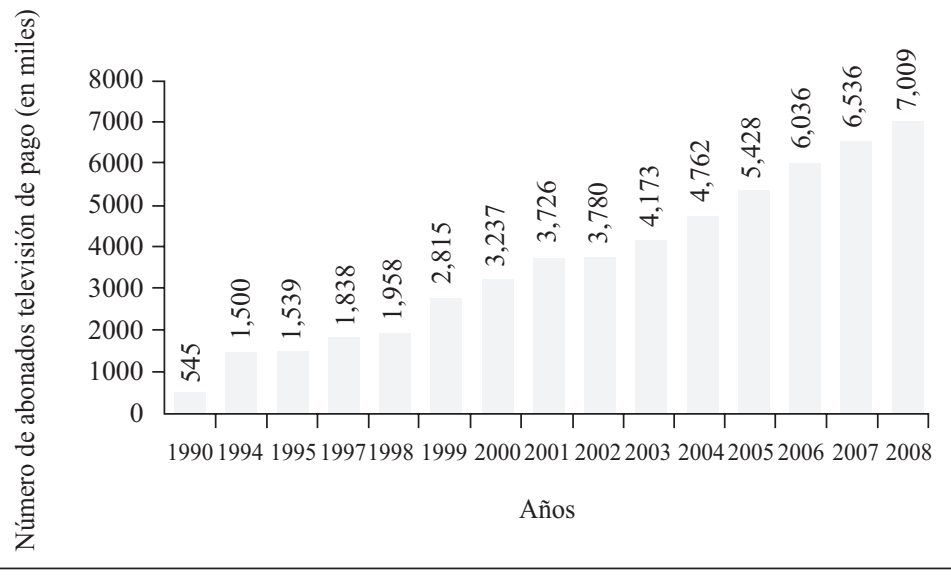

Fuente: Cofetel (2008).

Como consecuencia de este despunte inició un crecimiento ascendente en el número de abonados a las distintas plataformas, desde principios de la década de los noventa, como se aprecia en la Gráfica 2. Sin embargo, es importante establecer que en términos brutos, la televisión de paga todavía es un mercado minoritario, pues al término de 2008

15 DGTIE, Cofetel, con información de las empresas. 
apenas penetraba en $24.7 \%$ de los hogares en México, según el Instituto Nacional de Estadística, Geografía e Informática (INEGI). Esta cifra contrasta con $93.3 \%$ de los hogares con televisión abierta y con la penetración de la telefonía celular o móvil que en este país era, hasta el mismo año, de 70.3 líneas por cada 100 habitantes (Cofetel, 2009). Una nueva encuesta del INEGI reveló que al cierre de 2009, ya era 27.2\% (2.5\% más) de los hogares del país los que contaban con suscripción a los servicios de televisión por cable, satélite o microondas (Mejía, 2010).

El sistema que reportó hasta septiembre de 2009 más abonados es el de la televisión por cable, con 4'974,000 suscriptores. Estos se agrupan en la ahora denominada Cámara Nacional de la Industria de las Telecomunicaciones por Cable (Canitec), organismo que al igual que la Cámara Nacional de la Industria de la Radio y la Televisión (CIRT) se ocupa de las distintas acciones e iniciativas que toman en común los empresarios del subsector. 16 Por ejemplo, en materia tecnológica, de abastecimiento, de programación extranjera, de asesoría jurídica y, sobre todo, para actuar en bloque en la nivel político, para realizar acciones de cabildeo.

\section{CONVERGENCIA}

El futuro de la televisión de paga pasa actualmente por la incorporación de sistemas digitales que puedan ofrecer múltiples servicios, los cuales van desde la telecompra y la telefonía, pasando por la interactividad al seleccionar planos de eventos deportivos o de variedades con el mando a distancia, hasta la incorporación de Internet y la posibilidad de poder difundir productos audiovisuales realizados por los propios abonados (Gómez, 2002).

En 2006 fue publicado por la SCT el denominado "Acuerdo de Convergencia..." que establece las condiciones para que los concesionarios de cable puedan ofrecer el servicio de telefonía fija, conexión a Internet y distribución de contenidos (triple play, como ya se ha comentado) (Diario Oficial de la Federación 2006, 3 de octubre). En contraparte, a

16 Hasta 2007, Canitec significaba Cámara Nacional de la Industria de la Televisión por Cable. La posibilidad de prestar servicios adicionales les llevó a modificar su denominación. La organización fue fundada en 1975. 
las empresas telefónicas se les permite, a través de sus infraestructuras, la distribución de contenidos audiovisuales y de Internet. Telmex tiene en su título de concesión la prohibición expresa de ofrecer el servicio de televisión, por lo que el referido acuerdo no le garantiza la posibilidad de brindar el triple play (su oferta actual es sólo de telefonía e Internet).

Desde ese mismo año algunos operadores de cable comenzaron el despliegue de infraestructura para servicios triple play. Cablevisión, Megacable, Cablemás, Televisión Internacional (TVI), Telecable y Cablecom, empresas destacadas en el sector por número de clientes, son las que más han avanzado en el terreno de la convergencia. Durante 2008 captaron $28 \%$ del mercado de Internet de banda ancha en el país y alrededor de $2 \%$ de segmento de telefonía fija. Una consultoría mexicana estimó que $12 \%$ de los suscriptores de televisión por cable tienen el servicio de triple play (Mejía, 2009a). Otro dato relevante, más actualizado, nos muestra claramente la reconversión empresarial que viven las empresas de la televisión por cable: durante los primeros nueve meses de 2009, los ingresos de Megacable, Cablevisión y Cablemás por concepto de servicios de telefonía, acceso a Internet y redes contribuyeron con $36 \%$ de su facturación total, mientras que el servicio de televisión de paga aportó 59\% de sus ingresos totales (Vidal, 2010).

Según la Cofetel los beneficios para los usuarios residenciales con el triple play se han traducido en descuentos de hasta $49 \%$ en los precios del paquete, en comparación con la contratación por separado de estos servicios.

Pero aun con la fuerte reducción en los precios, hay estimaciones de consultoras especializadas como Select que consideran que los servicios triple play no llegarán a penetrar de manera importante al mercado masivo, toda vez que sólo alrededor de 7 millones de hogares, de casi 26 millones que hay en el país, son familias que ganan mas de siete salarios mínimos, es decir, con la capacidad económica para gastar 600 pesos al mes para estos paquetes (Mejía, 2008a).

No obstante esta situación, las inversiones se prevé seguirán incrementándose en los siguientes años, con el objetivo de modernizar y hacer más eficientes las redes de telecomunicación vía la digitalización. 
El problema de fondo que aquí planteamos es que la infraestructura tecnológica de última generación y que detona una serie de servicios y bienes relacionados con los productos culturales y la generación de conocimiento, están siendo guiadas, principalmente, por la lógica mercantil y no por el acceso universal o de servicio público. Este hecho genera mayores inequidades en un país tan desigual como México.

\section{COMPETENCIA Y CONCENTRACIÓN}

Otra característica que podemos establecer sobre la televisión de paga en México, es que a partir de la política de otorgamiento de concesiones por parte del gobierno y de la asociación de la mayoría de los concesionarios de cable, no se ha propiciado la competencia directa entre los distintos operadores en mercados tan importantes como el de la ciudad de México, donde Cablevisión de Televisa es el único prestador del servicio en esa plataforma. Además de este factor, el mercado de la televisión de paga está viviendo una de las concentraciones más intensas, producto de las adquisiciones que Grupo Televisa ha llevado a cabo en los últimos tres años (2006-2009).

El 29 de marzo de 2006, Televisa notificó a la CFC la adquisición, por casi 68 millones de dólares, de 49\% de Televisión Internacional (TVI, con presencia en Monterrey, estado de Nuevo León y su área conurbada) propiedad del Grupo Multimedios Estrellas de Oro. Casi un año después, el 21 de febrero de 2007, la empresa dio a conocer la inversión de 258 millones de dólares en obligaciones convertibles por $99.99 \%$ del capital social de Alvafig, empresa tenedora de 49\% de Cablemás (con sistemas en diversas localidades de Baja California, Campeche, Chihuahua, Guerrero, Hidalgo, Jalisco, Estado de México, Morelos, Oaxaca, Quintana Roo, San Luis Potosí, Tamaulipas, Veracruz y Yucatán).

El 29 de septiembre de 2006, la CFC emitió una resolución sobre la concentración de Televisa y TVI, la cual fue ampliada el 25 de febrero de 2007. Según el órgano antimonopolio, la transacción sólo sería autorizada si se cumplían las siguientes condiciones (CFC, 2007a):

Must offer: Grupo Televisa estará obligado a ofrecer sus contenidos de televisión abierta en condiciones no discriminatorias a todas aquellas 
empresas de televisión restringida que lo soliciten. Esta obligación será válida para todo el territorio nacional. Con ello se garantiza que los consumidores podrán acceder a la programación de Televisa aunque el operador de televisión restringida que contraten sea competidor de esta empresa; en otras palabras, se evita que los contenidos de Televisa impliquen una barrera para la entrada y el crecimiento de otros jugadores en el mercado de la televisión restringida.

Must carry: Las redes de televisión restringida de Grupo Televisa y TVI estarán obligadas a transmitir en condiciones no discriminatorias todos los contenidos de televisión abierta cuyos transmisores así lo soliciten. Esta obligación aplicará igualmente para el Grupo Televisa en toda la República. De esta manera, se asegura que los consumidores podrán tener acceso a todos los contenidos de televisión abierta -sean o no de Televisa-, nacionales y locales, sin importar que su proveedor de servicios de televisión restringida tenga ligas con esta empresa; dicho de otro modo, esta condición impide a las redes de televisión restringida de Grupo Televisa y TVI desplazar los contenidos de televisión abierta de otros oferentes (actuales o potenciales).

Independencia de redes de transmisión: Grupo Televisa y TVI deberán tomar acciones para impedir la coordinación con otras redes de telecomunicaciones que sean sus competidores actuales o potenciales. En particular, Grupo Televisa deberá prohibir que los miembros de sus consejos de administración participen en los órganos de administración y operación de otros concesionarios de redes, y TVI deberá enajenar sus acciones en PCTV, en la que es socio de otros concesionarios de televisión por cable.

El 12 de julio de 2007, la CFC resolvió la concentración entre Televisa y Cablemás, bajo prácticamente los mismos términos que condicionaron la adquisición de TVI, pero fortaleciendo algunos criterios como la obligatoriedad de Televisa de transmitir por las redes de televisión vía satélite aquellas señales que tengan una cobertura territorial de al menos 30\%. De igual manera, para los canales locales transmitidos a través de televisión vía satélite, se impuso a Televisa la obligación de 
carry one, carry all, que prohíbe discriminar a los concesionarios de televisión abierta local. 17

El pleno de la CFC decidió aprobar las operaciones - con las condiciones ya mencionadas- por las siguientes razones: a) Televisa es la única empresa para hacer un contrapeso a Telmex, cuando entre al mercado de video; b) en México el servicio de televisión por cable está muy pulverizado, por lo que se requiere tener a dos o tres empresas de buen tamaño para que puedan competir con la telefónica, y c) hay otras empresas de la televisión como que se están consolidando, como Megacable, la cual adquirió a Multioperadora de Sistemas, por lo que no hay problema de que Televisa tenga una presencia cada vez más relevante en el mercado de la televisión de paga (Gascón, 2007, 13 de agosto).

La CFC determinó que la concentración se haría efectiva hasta que Televisa acreditara haber cumplido la disposición de otorgar a todas aquellas empresas de televisión abierta o restringida que lo soliciten acceso a sus contenidos y a sus redes, en condiciones no discriminatorias. En los días siguientes, Televisa puso a disposición sus canales abiertos junto con otros canales que se ofrecen en sus sistemas de televisión de paga, pero lo hizo de manera empaquetada y estableciendo restricciones para que Telmex pudiera tener acceso a ellos. Aún así, cerca de 170 empresas de televisión por cable compraron los contenidos de Televisa y la CFC aprobó finalmente las concentraciones (Sosa, 2008a).

Con las adquisiciones, Televisa operaba, ya desde 2006, casi $50 \%$ de la televisión restringida en México (si se agregan los clientes de TVI, Cablevisión y Sky), con más de 3 millones de suscriptores (Tabla 1). Además de la concentración de clientes, Televisa se conviertió en el principal operador en 21 mercados de distribución y comercialización de paquetes de canales en televisión de paga de la República Mexicana. En 11 mercados era el único. 18

17 "En otras palabras, si Grupo Televisa decide transmitir una señal abierta local por sus redes de televisión vía satélite, tendrá la obligación de transmitir todas las señales de esta misma localidad, para evitar desventajas entre señales locales" (CFC, 2007b).

18 Dos de los cinco comisionados de la CFC se opusieron a la concentración Televisa-Cablemás. Uno de estos comisionados, Miguel Flores Barnés, pre- 
Adicionalmente a las adquisiciones de empresas de cable, Televisa compró en 2008 a Bestel, operador con la segunda red de telecomunicaciones de mayor tamaño en el país, con la cual fortalecerá sus servicios de triple play en el país. A este movimiento se suma su probable participación, durante 2010, en la licitación de bandas de frecuencias de 1.9 y $1.7 \mathrm{Ghz}$ del espectro radioeléctrico para prestar servicios de comunicaciones móviles y estar en condiciones de ofrecer servicios de cuádruple play (Internet, televisión, telefonía fija y telefonía móvil). El panorama para Televisa era alentador en esta licitación si se considera que mientras para los operadores de telefonía móvil (Telcel, Iusacell y Telefónica Movistar) se establecieron límites de acumulación de espectro en dichas bandas, la televisora incursiona en un terreno virgen y con amplias posibilidades de obtener las frecuencias para una red móvil a nivel nacional. En su objetivo de convertirse en un nuevo operador de servicios móviles, Televisa logró mediante un intenso cabildeo que el Congreso exentara a los ganadores de la licitación mencionada para ser librados del pago de derechos durante dos años (Sosa, 2009b, p. 27). A inicios de 2010, anunció la adquisición de 30\% de la telefónica y de servicio de trunking, Nextel de México, filial de NII Holdings, por 1,440 millones de dólares, para ir juntas en la lici-

sentó un voto razonado en el que expuso que de aprobarse la adquisición "Grupo Televisa adquirirá el poder de fijar unilateralmente los precios -en el mercado de suministro de canales de televisión y audio restringidoen los municipios de Camargo, Cuauhtémoc, Delicias y Meoqui, Chihuahua; Tepeji del Río Hidalgo; Lagos de Moreno y Ocotlán, Jalisco; Los Reyes Acaquilpan y Chimalhuacán, Estado de México; Chetumal y Mahahual, Quintana Roo; Ciudad Mante, Tamaulipas; Coatzintla y Minatitlán, Veracruz; Tecate y Playa de Rosarito, Baja California; Iguala y Zumpango del Río, Guerrero, y Tula de Allende, Hidalgo, por el simple hecho de que será el único vendedor (monopolio) de los servicios de televisión y audio restringido o, al menos, tendrá una participación en el mercado que le otorgará poder sustancial en dichas localidades". De igual manera, Televisa "podría desplazar indebidamente a otros agentes o podría impedir su acceso al mercado relevante, en virtud de que estaría en posibilidad de ejercer su poder o llevar a cabo prácticas monopólicas relativas" (Sosa, 2008b, p. 6). 
tación. Si no se gana la licitación, no hay operación, dijo el presidente de Televisa, Emilio Azcárraga. Este empresario así explicó la alianza:

Lo importante es que Televisa no sólo agrega el valor de los recursos económicos, sino que le agrega el valor de toda la mercadotecnia que vamos a lanzar, la publicidad para posicionar marcas y nuevos productos, y al tamaño que está detrás de Grupo Televisa como empresa. Pero sobre todo, una de las cosas que más le interesaban a ellos y a nosotros, es que Televisa tiene 3.5 millones de clientes de video, a través de las inversiones que hemos hecho en cable y en Sky. Esto va a permitir a Nextel ofrecer sus teléfonos celulares a todos estos suscriptores, que es el cuádruple play. A su vez, las empresas de cable y Sky podrán ofrecer telefonía móvil a través de Nextel. Vamos a abrir la gama de servicios que ofrecerá Nextel a 3.5 millones de clientes, y Nextel ofrecerá los servicios de voz, video y datos de cable y Sky a sus 3 millones de clientes (López, 2010, p. 22).

Otro de los operadores que ha llevado a cabo concentraciones relevantes es Megacable, de la familia Bours. Esta empresa adquirió en los últimos dos años entre 15 y 20 operadores de cable.

TABLA 1

PARTICIPACIÓN DE TELEVISA EN LA TELEVISIÓN RESTRINGIDA DE MÉXICO 2006 (CIFRAS EN MILES DE SUSCRIPTORES)

\begin{tabular}{lcc} 
& Situación en 2006 & Con Cablemás y TVI \\
\hline Sky (Televisa) & 1,430 & 1,430 \\
Cablevisión (Televisa) & 496 & 496 \\
Cablemás & & 629 \\
TV Internacional & & 144 \\
Total Televisa & 1,926 & 2,699 \\
\hline Total industria & 5,576 & 5,576 \\
Participación de Televisa & $34.5 \%$ & $48.3 \%$ \\
\hline Hogares INEGI & 24,562 & 24,562 \\
Participación de hogares & $7.8 \%$ & $22.7 \%$ \\
\hline
\end{tabular}

Fuente: Elaboración propia con información de Informe Anual de Televisa 2007, INEGI. 


\section{Televisión por cable y MMDS}

Las empresas más importantes de la televisión por cable, por número de abonados, eran hasta 2007 Megacable al reportar 1'300,000; Cablemás con 797,000; Cablecom con 610,000 y Cablevisión con 551,000 abonados.

En MMDS, Multivisión, aglutinó alrededor de 552,000 suscriptores en 2008. Finalmente, la televisión directa al hogar o DTH, controlada por Grupo Televisa a través de Sky, concluyó en 2008 con cerca de 1' 500,000 abonados.

Como hemos comentado, la mayoría de los operadores de sistemas de televisión por cable se agrupan en PCTV (96\%). Algunas de las empresas más importantes en esta agrupación son: Megacable, Cablemás, Hi! Telecomunicaciones, Televicable, Telecable, Cota Castro, Televicable del Centro, TVI, Televisión por Cable del Norte de Sonora, MATSA, Controladora de los Altos, FRAVI, Corporativo Núcleo Radio TV, Cable de Tuxtla, Imatel Comunicaciones, RGT e IRA Consultores Administrativos (www.canitec.org).

\section{GRÁFICA 3}

DISTRIBUCIÓN POR ABONADOS DE LAS PRINCIPALES EMPRESAS DE TELEVISIÓN DE PAGA (EN PORCENTAJES)

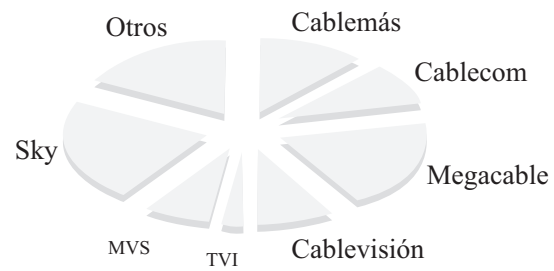

Fuente: Elaboración propia con datos de las empresas y la Cofetel.

Como ya habíamos advertido, las señales y contenidos que ofrecen las distintas empresas de televisión de paga son similares entre ellas. En el caso de la televisión por cable habría que agregar que las pocas diferencias obedecen a cuestiones puntuales de tipo moral, que van en función de los dueños de la empresa y de los estados en donde ofrecen sus 
servicios. ${ }^{19}$ Otra diferencia la identificamos en el ámbito económico, dependiendo del respaldo financiero que tienen las distintas empresas para tender los nuevos cableados de fibra óptica o coaxiales híbridos, que son necesarios para ofrecer Internet por medio de la televisión y telefonía fija a través de los cables, lo que las coloca en una posición privilegiada frente al DTH y el MMDS.

Finalmente, observamos una participación de capitales estadounidenses en las compañías más importantes del sector. ${ }^{20}$

En MMDS el principal operador es Multivisión. La segunda empresa en importancia es Ultravisión, de la familia Zorrilla, con operaciones en Puebla y Veracruz.

\section{Televisión directa al hogar o DTH}

Esta es la última modalidad de televisión de paga que se ha incorporado en la industria televisiva. En el mercado latinoamericano, incluido México, surgieron, como se detalló antes, dos empresas denominadas DirecTV Latin América y Sky Latin América. Las diferencias entre ambas plataformas eran mínimas, pues ofrecían casi los mismos canales. Una de las pocas diferencias era la compra de derechos de eventos especiales y deportivos para su difusión en exclusiva. Por ejemplo, Directv compró los derechos de los mundiales de Corea y Japón 2002 y Alema-

19 Por ejemplo, la empresa Cablemás dentro de su oferta de canales tiene contemplado al canal Playboy, mientras que Telecable que opera en estados conservadores como Jalisco, Sonora y Sinaloa no ofrece esa señal.

20 En noviembre de 1993, Telecab (de Alejandro Álvarez Guerrero) recibió asistencia técnica por 8 millones de dólares con la firma estadounidense Kleven para el desarrollo de la televisión interactiva. También se alió con la Falcon Cable TV, Hellman \& Friedan y la Duhamel Broadcasting Enterprises para invertir 100 millones de dólares entre 1994 y 1998 para la instalación de fibra óptica en los sistemas de Telecab de Tijuana, Mexicali, Ciudad Juárez y Chihuahua. Un año después, en noviembre de 1994, Telecable Mexicano (Abraham Kaham) se asocia con la United International Holdings, la cual adquiere en mayo de 1995, 49\% de las acciones de la empresa. Esta asociación tuvo también como propósito instalar fibra óptica en los sistemas de Acapulco, Cuernavaca, Chilpancingo y Oaxaca. 
nia 2006 para la mayoría de los países latinoamericanos y ofrecía todos los partidos de la liga de fútbol americano profesional NFL. En México, la otra diferencia fue que nunca pudo retransmitir las señales en abierto de la empresa Televisa, porque ésta es accionista de Sky.

El crecimiento de los abonados de ambas plataformas fue constante desde su surgimiento en 1996, llegando casi a 600,000 en el año 2000 y alcanzando 1'200,000 en 2005, pero ya entonces controlados sólo por Sky al absorber, luego de su cierre de operaciones en el país, a los abonados de DirecTV a finales de 2004. Entre 2007 y 2008, el servicio DTH creció 7.8\% para llegar a 1'500,000 abonados en todo el país. De 2008 a septiembre de 2009, este incremento fue de $32 \%$ (su mayor tasa de crecimiento en los últimos ocho años) para llegar a poco más de 2 millones de suscriptores (Cofetel, 2009).

Como señalamos en el apartado anterior, durante 2008 se incorporó una nueva compañía de DTH: Dish México. Con este movimiento se recompone el mercado de la televisión de paga en general y rompe la posición monopólica de Televisa en DTH a través de Sky.

\section{ÍNDICES DE CONCENTRACIÓN DE MERCADO}

Además de las concentraciones llevadas a cabo mediante la adquisición de empresas de televisión por cable, el índice de concentración se puede medir a través del coeficiente de facturación y de suscriptores de las cuatro empresas dominantes (CR4). Este índice nos permite aportar elementos para observar la concentración del sector de forma cuantitativa y gráfica en términos económicos para caracterizar la estructura de mercado.

El índice contempla una escala de 0 a 1 punto, en donde por debajo de .2 se considera que en el mercado se desarrolla una "competencia perfecta". Entre .2 y .6 hay una competencia monopólica. Por arriba de 6 y menos de .8 hablamos de una estructura oligopólica, y finalmente entre $.8 \mathrm{y}$ el entero es un monopolio.

Para el caso del coeficiente de facturación tomamos en cuenta los ingresos brutos totales reportados por las cuatro empresas dominantes del sector y se contrastó con los ingresos brutos totales de todo el subsector de la televisión restringida reportado por la Cofetel en 2007. 
Según este índice las cuatro empresas dominantes (véanse Tabla 2 y Gráfica 4) aglutinan $68 \%$ del total de la facturación, lo cual arroja que el mercado de la televisión restringida, al ser de .68, está altamente concentrado y se caracteriza por tener una estructura oligopólica.

En el caso de los abonados la tendencia se reduce un poco, pero no contrasta con el resultado anterior, pues las cuatro empresas dominantes concentran $64 \%$ de los suscriptores en el nivel nacional. Esto reconfirma la estructura oligopólica del sector.

\section{TABLA 2}

NÚMERO DE ABONADOS E INGRESOS TOTALES DE LAS 4 EMPRESAS DOMINANTES DEL MERCADO DE LA TELEVISIÓN RESTRINGIDA EN MÉXICO 2007

\begin{tabular}{lrrcc} 
Empresa & Abonados & Porcentaje & $\begin{array}{c}\text { Facturación } \\
\text { (en miles de } \\
\text { pesos) }\end{array}$ & Porcentaje \\
\hline Cablemas & 797,018 & 12 & 2,703 & 10.6 \\
Cablevisión & 551,400 & 8 & 2,059 & 8.1 \\
Megacable & 1 '300,000 & 21 & 4,810 & 18.9 \\
SKY & $1 ' 482,000$ & 23 & 7,732 & 30.4 \\
Otros & 2 '‘05,782 & 36 & 8,041 & 32 \\
Total & $6 ' 536,200$ & 100 & 25,345 & 100 \\
\hline
\end{tabular}

Fuente: Elaboración propia con base en datos de los informes anuales de las empresas 2007 y la Cofetel.

GRÁFICA 4

COEFICIENTE DE CONCENTRACIÓN CR4 DEL MERCADO

DE LA TELEVISIÓN DE PAGA EN MÉXICO 2007

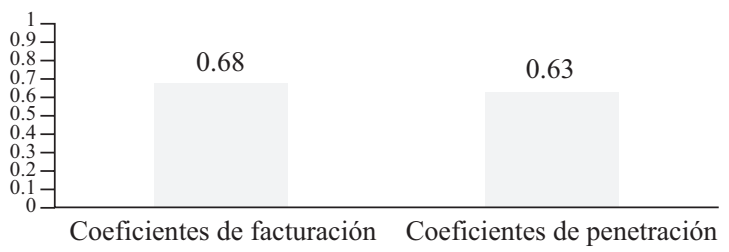

Fuente: Elaboración propia con base en datos de la investigación. 


\section{COBERTURA Y PENETRACIÓN}

Como se mencionó antes, la televisión de paga todavía es un mercado minoritario en México. Al término de 2008 apenas penetraba en 24.7\% de los hogares en el país, según el Instituto Nacional de Estadística, Geografía e Informática (INEGI). Esto significa que hasta ese mismo año había en promedio 66 suscriptores por cada 1,000 habitantes. Al analizar la penetración del servicio en cada uno de los estados del país, las diferencias de acceso se hacen más evidentes, como consecuencia no sólo de la cobertura sino también por las brechas económicas regionales y el poder adquisitivo de los mexicanos. Los estados con mayor número de suscriptores por cada mil habitantes son Baja California Sur (128), Quintana Roo (110) y Colima (107), mientras que las entidades que registran menos suscriptores en proporción a su población son las que históricamente se han mantenido en una situación de marginación, como Chiapas (24) y Oaxaca (29). Por participación porcentual, tres entidades, el Distrito Federal, Jalisco y el Estado de México, concentran la tercera parte del mercado nacional.

\section{TABLA 3}

SUSCRIPTORES DE TELEVISIÓN DE TELEVISIÓN RESTRINGIDA POR ENTIDAD FEDERATIVA.

DICIEMBRE 2008

Estado

\section{Suscriptores}

\section{Participación} porcentual
Suscriptores por cada 1,000 habi-

tantes

\begin{tabular}{lrrr}
\hline Nacional & $7{ }^{\prime} 053,934$ & 100 & 65.8 \\
Aguascalientes & 81,522 & 1.2 & 71.9 \\
Baja California & 269,288 & 3.8 & 86.2 \\
Baja California Sur & 71,328 & 1.0 & 127.7 \\
Campeche & 73,998 & 1.0 & 93.5 \\
Coahuila & 179,526 & 2.5 & 68.6 \\
Colima & 63,887 & 0.9 & 107.0 \\
Chiapas & 106,233 & 1.5 & 23.7 \\
Chihuahua & 207,240 & 2.9 & 61.4
\end{tabular}




\begin{tabular}{|c|c|c|c|}
\hline Estado & Suscriptores & $\begin{array}{c}\text { Participación } \\
\text { porcentual }\end{array}$ & $\begin{array}{l}\text { Suscriptores por } \\
\text { cada } 1,000 \text { habi- } \\
\text { tantes }\end{array}$ \\
\hline Distrito Federal & $1 ' 170,082$ & 16.6 & 71.9 \\
\hline Durango & 70,273 & 1.0 & 45.4 \\
\hline Guanajuato & 263,739 & 3.7 & 52.4 \\
\hline Guerrero & 143,673 & 2.0 & 45.7 \\
\hline Hidalgo & 144,047 & 2.0 & 59.6 \\
\hline Jalisco & 616,368 & 8.7 & 88.2 \\
\hline México & 519,965 & 7.4 & 71.1 \\
\hline Michoacán & 273,759 & 3.9 & 68.9 \\
\hline Morelos & 139,973 & 2.0 & 83.9 \\
\hline Nayarit & 71,131 & 1.0 & 73.5 \\
\hline Nuevo León & 366,252 & 5.2 & 82.8 \\
\hline Oaxaca & 104,265 & 1.5 & 29.4 \\
\hline Puebla & 227,052 & 3.2 & 40.4 \\
\hline Querétaro & 156,594 & 2.2 & 91.8 \\
\hline Quintana Roo & 142,343 & 2.0 & 110.3 \\
\hline San Luis Potosí & 150,572 & 2.1 & 60.7 \\
\hline Sinaloa & 189,477 & 2.7 & 71.5 \\
\hline Sonora & 234,628 & 3.3 & 93.9 \\
\hline Tabasco & 93,570 & 1.3 & 45.7 \\
\hline Tamaulipas & 269,267 & 3.8 & 84.8 \\
\hline Tlaxcala & 61,658 & 0.9 & 54.7 \\
\hline Veracruz & 365,739 & 5.5 & 53.1 \\
\hline Yucatán & 137,904 & 2.0 & 72.2 \\
\hline Zacatecas & 68,581 & 1.0 & 49.7 \\
\hline
\end{tabular}

Fuente: Cofetel.

Tradicionalmente, la oferta de los servicios de televisión de paga se ha caracterizado por estar dirigida a los hogares de alto poder adquisitivo, por lo que existe un amplio sector de la población no atendido. La entrada de Dish al mercado mexicano con un paquete básico de 25 canales -además de los canales culturales abiertos: 11, del Instituto Politécnico Nacional (IPN) y 22, adscrito al Consejo Nacional para la Cultura y las Artes- cuyo costo mensual es de 149 pesos mensuales 
(alrededor de 12 dólares estadounidenses), podría mejorar los índices penetración del servicio. Su estrategia de mercado también fomentó una disminución de precios que ofrecen algunos de sus competidores. ${ }^{21}$

Sin embargo, habrá un sector amplio de la población que no podrá tener acceso a ese servicio por las condiciones económicas del país. Según el INEGI, en 2008, 60\% de los mexicanos con menos dinero concentró 26.7\% del ingreso (por debajo de 27.6\% que tenían en 2006), mientras que $20 \%$ con más recursos pasó de $35.7 \%$ hace tres años a $36.3 \%$ del anterior. Es decir, se incrementaron las diferencias de ingreso. La Encuesta Nacional de Ingresos y Gastos en los Hogares (ENIGH) indica que el ingreso promedio trimestral en poblaciones de más de 2,500 habitantes es de 41,341 pesos (3,108 dólares) mientras que en los que tienen menos habitantes cae a 18,276 pesos (1,395 dólares).

Dividiendo en diez grupos a la gente por su nivel de ingresos el estudio indica que los que menos dinero obtienen, 6 mil 116 pesos por trimestre (466 dólares), vieron caer sus ingresos un $8 \%$, lo que representa el mayor descenso (EFE, 2009).

El gasto se concentró en alimentos, bebidas y tabaco (33.6\%), seguido por transporte (18.4\%), educación y esparcimiento $(13.5 \%)$, y vivienda y combustibles (10\%).

La misma ENIGH reveló que las familias mexicanas que usan servicios de comunicaciones gastan en promedio casi 492 pesos mensuales para pagarlos, monto superior en casi 14\% al que erogaban cuatro años antes. De esta manera, en los hogares se ha dejado de gastar en otros rubros, como cuidados de la salud y entretenimiento (cine o teatro, por ejemplo), para destinar recursos al consumo de telefonía, Internet y televisión de paga. Las familias con mayor poder adquisitivo gastaron en promedio 1,155 pesos al mes en dichos servicios, mientras que las de menor nivel socioeco-

21 Los principales operadores de televisión por cable: Cablevisión, TVI, Cablemás y Megacable crearon una alianza para ofrecer en 2009 un servicio de triple play denominado Yoo, a un precio de 499 pesos mensuales (alrededor de 38 dólares estadounidenses). También la empresa Sky modificó sus tarifas comerciales (Mejía, 2009b). 
nómico consumieron 448 pesos al mes (Mejía, 2009c). Parte importante de este gasto se destinó, en primer término, a la telefonía celular, según la Profeco, si se considera la enorme penetración de este servicio. Le siguen otros servicios (Publimetro, 2009).

\section{CONSIDERACIONES FINALES Y DISCUSIÓN}

Las tendencias en México parecen ser claras. Operadores de la televisión por cable y el DTH seguirán compitiendo por los consumidores de estratos socioeconómicos medios y altos, utilizando toda la potencialidad que les ofrece las nuevas tecnologías a las plataformas digitales, lo que seguramente repercutirá en más fusiones entre algunas compañías de cable, para hacerle frente a las concentraciones encabezadas por Grupo Televisa. La posibilidad, postergada en los últimos años, para que Telmex se incorpore al mercado de la televisión restringida, vía sus infraestructuras, traería en teoría un posible beneficio a los consumidores en términos de precios, sin embargo, en cuanto a la oferta de los contenidos los cambios serán, a nuestro parecer, mínimos y no se estará propiciando una mayor diversidad y pluralidad ni tampoco en un impulso decidido a la producción nacional audiovisual. ${ }^{22}$

Por su parte, Multivisión a través de su servicio MasTV23 y, ahora, con Dish México, apuesta por los consumidores de poder adquisitivo bajo; situación que muestra su estrategia de captar amplios sectores de

22 Telmex incursionaría en televisión restringida a través de la tecnología IPTV o Televisión sobre Protocolo de Internet. En caso de que esta empresa que detenta $70 \%$ del mercado de telefonía fija ofreciera televisión de paga, en 2012 habría 2.2 millones de suscriptores del servicio IPTV, según estimaciones de Frost and Sullivan. En México, sólo una empresa, Maxcom, ofrece el servicio de IPTV. "TV de paga, aún con poca competencia”, en El Universal (2009, 21 de julio, p. B-8).

23 El servicio de televisión de Multivisión o MVS cambió por el nombre de MasTV en el año 2002. La modificación de su denominación también implicó una reducción de la oferta de canales y de precio. Actualmente la oferta de MasTV es de sólo 15 canales, igual cantidad con la que ahora Dish México ofrece su paquete básico en la plataforma DTH (Sosa, 2009a). 
la sociedad mexicana. La táctica de la empresa de canalizar a sus suscriptores de MMDS a la tecnología satelital, ha llevado a que la televisión por microondas presente signos de desaceleración. El número de usuarios de esta tecnología registró su séptima disminución anual desde el cierre de 2007 al caer 21.1\% durante el tercer trimestre de 2009, el mayor tropiezo desde finales de 2002, lo que ocasionó que el número de suscriptores cerrara en 565,000 (septiembre 2009). En cambio, DTH tendrá un incremento en los meses y años venideros si los resultados y previsiones anunciadas por Dish México se confirman. ${ }^{24}$

También, como hemos observado, la televisión de paga en México refuerza la tendencia a la concentración de los grandes grupos mediáticos, los mismos que operan en los distintos sectores de la industria audiovisual, situación que limita la oferta que ofrecen estos sistemas $\mathrm{y}$, sobre todo, la pluralidad en la distribución de las señales. Bajo tal panorama, la tan anunciada competencia sólo es parte de la retórica discursiva de las últimas administraciones neoliberales, manifestada a través de los programas de gobierno (como el Programa Sectorial de Comunicaciones y Transportes 2007-2012, de la SCT). Así mismo se advierte una participación cada vez más constante de capitales estadounidenses en los distintos sistemas de televisión de paga. Esta tendencia seguramente se reforzará al necesitarse más inversiones para hacer frente al escenario de competencia que se avecina y a la innovación tecnológica, así como la digitalización, sobre todo en una situación de desaceleración por la crisis económica que se presenta desde finales de 2008 .

De igual manera, como consecuencia de la crisis económica y ante la falta de políticas públicas y de una legislación que estimule la producción independiente y proteja a la nacional, los sistemas de la televisión de paga continuarán desarrollándose como simples difusores de señales de contenidos de origen estadounidense, lo cual genera otras

24 “(En) julio de 2009, Dish contaba con 530,000 suscriptores. Al final de año anticipaba llegar al millón de clientes y cubrir 16 ciudades del país" (Reuters, 2009, 20 de agosto: "MVS podría invertir 700 mdd"). Al cierre de este artículo, diversos columnistas de negocios, confirmaron que la meta se había cumplido, pero la empresa no lo había notificado oficialmente. 
repercusiones en el ámbito de las industrias culturales que deberán analizarse con más detalle.

Finalmente, hacemos hincapié en señalar que la forma en la cual se está desarrollando el mercado de la televisión de pago y sus servicios agregados como la banda ancha, es sólo bajo la lógica mercantil, situación que en sí misma excluye a un gran número de mexicanos que no serán considerados por su poco poder adquisitivo. Este hecho seguirá acrecentando desigualdades y contradicciones en materia de acceso, apropiación y consumos relacionados, tanto con la cultura como con la generación de conocimiento. Por lo tanto, es imperativo que en paralelo a este desarrollo, desde el poder público, a través de políticas públicas con sentido de servicio público, presente alternativas de inclusión a esos grandes sectores de la población que están siendo excluidos.

\section{Bibliografía}

Becerra, M. \& Mastrini, G. (2006). Periodistas y magnates. Estructura y concentración de las industrias culturales en América Latina. Buenos Aires: Prometeo.

Bouquillion, P., Miege, B. \& Moriset, C. (2006). Concentration capitalistique dans les industries culturelles et médiatiques. Le Temps des Médias, 6, pp. 151-164.

Bustamante, E. (2003). Las industrias culturales entre dos siglos. En E. Bustamante (Coord.), Hacia una nuevo sistema mundial de comunicación. Las industrias culturales en la era digital (pp. 19-38). Barcelona: Gedisa.

CFC-Comisión Federal de Competencia (2006). Expediente DE-012006. Recuperado el 15 de septiembre de 2009 de http://www.cfc. org.mx

CFC-Comisión Federal de Competencia (2007a). Comunicado 02-2007. Recuperado el 18 de marzo de 2009 de http://201.161.75/Index. php?option $=$ com_content\&trask=view\&id=3609\&ltemid $=204$

CFC-Comisión Federal de Competencia (2007b). Comunicado 06-2007.

Recuperado el 18 de marzo de 2009 de http://201.161.46.75/index. php?option $=$ com_content\&trask=view\&id=3609\&Itemid=204

Cofetel-Comisión Federal de Telecomunicaciones (2003). Dirección General de Televisión y Audio Restringidos. Febrero. México. 
Cofetel-Comisión Federal de Telecomunicaciones (2008). Televisión y audio restringidos. Recuperado el 20 de septiembre de 2008 de http://www.cofetel.gob.mx/wb/Cofetel-2008/Cofete_television_y_ audio_restringidos.

Cofetel-Comisión Federal de Telecomunicaciones (2009). Índice de Producción del Sector Telecomunicaciones. Tercer trimestre. Recuperado el 26 de febrero de 2010 de http:/www.cofetel.gob.mx/wb/ cofetel-2008/tercer_trimestre_2009

Crovi, D. (1999). Televisión por cable en México. Una industria en busca de nuevos rumbos. Comunicación y Sociedad, 35, 131-150.

Crovi, D. (2005). De la televisión por cable a las redes públicas de telecomunicaciones 50 años de CATV en México. En L. Martell, M. Rizo \& A. Vega (Eds.), (2005). Políticas de comunicación social y desarrollo regional en América Latina (pp. 87-126). Vol. 1. México: $\mathrm{UACM} / \mathrm{AMIC}$.

Diario Oficial de la Federación (1993, 23 de agosto). Decreto por el que se reforman los artículos 10, 33 y 42 del Reglamento del Servicio de Televisión por Cable. Recuperado el 25 de marzo de 2009 de http://dof.gob.mx/index.php?year=1993\&mont=08\&day=23

Diario Oficial de la Federación (2006, 3 de octubre). Acuerdo de convergencia de servicios fijos de telefonía local y/o audio restringidos que se proporciona a través de redes públicas alámbricas e inalámbricas. Recuperado el 25 de marzo de 2009 de http://www.sct. gob.mx/fileadmin/normatividad/telecomunicaciones/2010/102\%20 Acuerdo $\% 20$ de $\% 20$ convergencia $\% 20$ de $\% 20$ servicios $\% 20$ fijos $\% 20$ de $\% 20$ telefon $\%$ EDa $\% 201$ local $\% 20 y \% 20$ televisi $\%$ F3n $\% 20$ yo $\% 20$ audio $\% 20$ restringidos $\% 20$ que $\% 20$ se $\% 20$ proporcionan $\% 20$ a $\% 20$ trav\%E9s\%20de\%20re.pdf

EFE, Agencia de noticias (2009, 17 de julio). Desigualdad entre los mexicanos más ricos y los más pobres aumentó en 2008. El Periódico de México. Recuperado el 25 de julio de 2009 de http://www. elperiodicodemexico.com/notaphp?id=271232

El Universal (2009, 21 de julio). TV de paga, aún con poca competencia. Finanzas.

Garnham, N. (2000). Emancipation, the media and modernity. Arguments about media and social theory. Nueva York: Oxford University Press. 
Garnham, N. (2005). From cultural to creative industries. An analisys of the implications of the "creative industries" approch to arts and media policy making in the United Kingdom. International Journal of Cultural Policy, 1. Vol. 11. Londres: Routledge, 15-29.

Gascón, V. (2007, 13 de agosto). Niegan dominio de Televisa en Reforma. Recuperado el 25 de marzo de 2009 de http://www.cencos.org/ ees/node/16696

Golding, P. \& Murdock, G. (2000). Culture, communications, and political economy. En J. Curran \& M. Gurevitch, Mass media and society (pp. 15-52). Londres: Edward Arnold.

Gómez, R. (2002). Convergencias mediáticas. Una propuesta para su estudio. Comunicación presentada en el IV Congreso Internacional de Comunicación, efectuado en la Universidad Pontificia de Salamanca. España.

Gómez, R. \& Sosa, G. (2006). Reforma de la legislació en radio, televisió i telecomunicacions a Méxic. Quaderns del CAC, 25, 63-80. Barcelona, Catalunya.

Gómez, R. (2007). Políticas de comunicación en México. El giro neoliberal, 1988-2006. En Rodrigo G. \& Adriana P. Comunicación para el desarrollo (pp. 389-429). Morelia: AMIC.

Herman, E. \& McChesney, R. (1997). The global media. The new missionaries of corporate capitalism. Londres: Casell.

Hernández, F. \& Orozco, G. (2007). Televisiones en México. Un recuento histórico. México: UdeG.

Hoskins, McFadyen \& Finn (2004). Media economics:applying economicas to new and traditional media. Londres: Sage.

INEGI-Instituto Nacional de Estadística, Geografía e Informática (2006). Información estadísitca, medio de comunicación. Recuperado el 20 de abril de 2008 de http://www.inegi.org.mx/est/contenidos/espanol/soc/sis/sisept/default.aspx?+=mcul24\&S=est\&c=5709

Iosifides, P. (1997). Methods of measuring media concentration. Media, Culture \& Society, Vol. 19. Londres: Sage, 643-663.

López, J.F. (2010, 26 de febrero). Nextel. Entrevista a Emilio Azcárraga. Poder y Negocios. Recuperado el 10 de agosto de 2009 de http:// www.eluniversal.com.mx/Finanzas/71879.html 
Mejía, A. (2008). Triple play impulsa la competencia. El Universal. Memoria 2000. Recuperado el 20 de marzo de 2009 de http:/www.eluniversal.com.mx/graficos/00coberturas/anuario2008/uno-cinco.html

Mejía, A. (2009a, 3 de agosto). Atrae a población de bajos ingresos oferte "triple play". El Universal. Finanzas.

Mejía, A. (2009b, 29 de junio). Guerra de tarifas en la TV de paga. El Universal. Finanzas.

Mejía, A. (2009c, 24 de agosto). Mexicanos gastan más telecomunicaciones. El Universal. Finanzas. Recuperado el 15 de septiembre de 2009 de http://www.eluniversal.com.mx/Finanzas/73072.html.

Mejía, A. (2010, 22 de febrero). Triple play alienta avance de TV de paga. El Universal. Cartera. Recuperado el 26 de febrero de 2010 de http://www.eluniversal.com.mx/finanzas/77572.html

Miège, B. (2006). La concentración en las industrias culturales y mediáticas (ICM) y los cambios en los contenidos. CIC Cuadernos de Información y Comunicación, Vol. II. Madrid, 155-166.

Miguel, J. C. (1993). Los grupos multimedia: estructura y estrategias en los medios europeos. Barcelona: Bosch.

Mosco, V. (1996). The political economy of communication. Londres: Sage.

Murdock, G. (2006). Bajo la playa, los adoquines: mercancias, consumismo, contradicciones. CIC Cuadernos de Información y Comunicación, Vol. II. Madrid, 31-46.

Publimetro (2009). Gastan mexicanos hasta 20 por ciento de su ingreso en celular. Recuperado el 8 de julio de 2009 de http://www.publimetro.com.mx/noticias/gastan-mexicanos-hasta-20-por-ciento-de-suingreso-en-celular/pigh'ynDbf5r6dih8ZOflbC2ARw/

Profeco (2001). Profeco realiza estudio de mercado sobre televisión por cable. Comunicado 29 junio de 2001. Recuperado el 25 de marzo de 2009 de http://www.profeco.gob.mx/prensa/prensa01/ jun01/025bol_29062001.pdf

Reuters (2009, 20 de agosto). Dish espera llegar al millón de clientes. CNN Expansión.com, Recuperada el 16 de septiembre de 2009, de http://cache.cnnexpansion.com/actualidad/2009/08/20/dish-esperaduplicar-clientela 
Sánchez, E. (2006). Industrias culturales, diversidad y pluralismo en América Latina. CIC Cuadernos de Información y Comunicación, Vol. 11. Madrid, 207-221.

SCT-Secretaría de Comunicaciones y Transportes (2007). Programa Sectorial de Comunicaciones y Transportes 2007-2012. Recuperado el 20 de agosto de 2009 de http://www.sct.gob.mx/uploads/media/ SCT_PS_2007-2012.pdf

Sevilla, P. (1998). Del Cable al DTH. En Sánchez de Armas (Coord.), Apuntes para una historia de la televisión mexicana (pp. 415-484). México: Revista Mexicana de Comunicación/Televisa.

Sosa, G. (2008a, 20 de mayo). Fortalecer a Televisa para debilitar a Telmex. El Universal. Finanzas. Columna Telecom y Medios. Recuperado el 10 de septiembre de 2009 de http://www.eluniversal.com. $\mathrm{mx} /$ columnas/71497.html.

Sosa, G. (2008b, 27 de mayo). El voto contra la concentración TelevisaCabkenpas. El Universal. Finanzas. Columna Telecom y Medios. Recuperado de el 11 de septiembre de 2009 de http://www.eluniversal.com.mx/columas/71619.html.

Sosa, G. (2009a, 20 de enero). MVs: concesiones en disputa. El Universal. Finanzas. Columna Telecom y Medios. Recuperada el 24 de marzo de 2009 de http://www.wl-universal.com.mx/columnas/76376.html

Sosa, G. (2009b, 27 de noviembre). Próxima licitación: ¿más amparos en puerta?. El Universal. Cartera. Recuperado el 26 de enero de $2010 \mathrm{de} \mathrm{http://www.eluniversal.com.mx/editoriales/46471.html}$

Telemundo (2008). Núm. 103. Octubre-noviembre. Recuperado el 25 de enero de 2009 de http://www.canal100.com.mx/telemundo/ informes/?id_hoja $=783$

Televisa (2007) Informe Anual 2007. México: Televisa.

Vidal, F. (2010). Los efectos de la crisis. Revista Mexicana de Comunicación, 120. Febrero-abril. Recuperado el 10 de enero de 2010 de http://www.mexicanadecomunicacion.com.mx/mc120_1.thml 